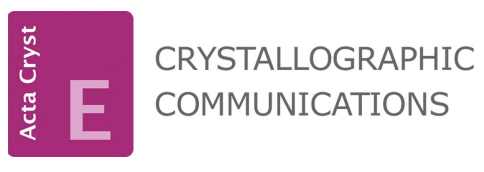

ISSN 2056-9890

Received 1 July 2017

Accepted 21 August 2017

Edited by M. Zeller, Purdue University, USA

Keywords: crystal structure; manganese; pyridine $\mathrm{N}$-oxide ligand.

CCDC references: 1570001; 1570000 ; 1569999

Supporting information: this article has supporting information at journals.iucr.org/e

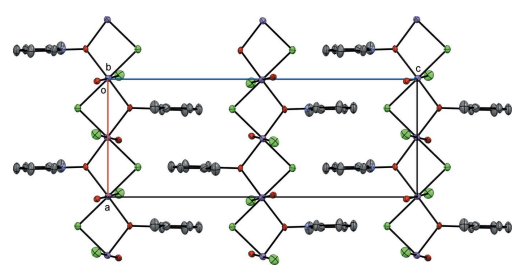

OPEN $\odot$ ACCESS

\section{Manganese(II) chloride complexes with pyridine $N$-oxide (PNO) derivatives and their solid-state structures}

\author{
Linda Kang, ${ }^{a}$ Genevieve Lynch, ${ }^{b} *$ Will Lynch ${ }^{a}$ and Clifford Padgett ${ }^{a}$ \\ ${ }^{a}$ Department of Chemistry and Physics, Armstrong State University, Savannah, Georgia 31419, USA, and ${ }^{\mathbf{b}}$ St Vincent's \\ Academy, Savannah, Georgia 31401, USA. *Correspondence e-mail: will.lynch@armstrong.edu
}

Three manganese(II) $N$-oxide complexes have been synthesized from the reaction of manganese(II) chloride with either pyridine $N$-oxide (PNO), 2-methylpyridine $\mathrm{N}$-oxide (2MePNO) or 3-methylpyridine $\mathrm{N}$-oxide (3MePNO). The compounds were synthesized from methanolic solutions of $\mathrm{MnCl}_{2} \cdot 4 \mathrm{H}_{2} \mathrm{O}$ and the respective $\mathrm{N}$-oxide, and subsequently characterized structurally by single-crystal X-ray diffraction. The compounds are catena-poly[[aquachloridomanganese(II)]-di- $\mu$ chlorido-[aquachloridomanganese(II)]-bis $\left(\mu\right.$-pyridine $N$-oxide)], $\left[\mathrm{MnCl}_{2}\left(\mathrm{C}_{5} \mathrm{H}_{5^{-}}\right.\right.$ $\left.\mathrm{NO})\left(\mathrm{H}_{2} \mathrm{O}\right)\right]_{n}$ or $\left[\mathrm{MnCl}_{2}(\mathrm{PNO})\left(\mathrm{H}_{2} \mathrm{O}\right)\right]_{n}(\mathbf{I})$, catena-poly[[aquachloridomanganese(II)]-di- $\mu$-chlorido-[aquachloridomanganese(II)]-bis $(\mu$-2-methylpyridine $N$-oxide $)],\left[\mathrm{MnCl}_{2}\left(\mathrm{C}_{6} \mathrm{H}_{7} \mathrm{NO}\right)\left(\mathrm{H}_{2} \mathrm{O}\right)\right]_{n}$ or $\left[\mathrm{MnCl}_{2}(2 \mathrm{MePNO})\left(\mathrm{H}_{2} \mathrm{O}\right)\right]_{n}(\mathbf{I I})$, and bis( $\mu$-3-methylpyridine $N$-oxide)bis[diaquadichloridomanganese(II)], $\left[\mathrm{Mn}_{2} \mathrm{Cl}_{4}\right.$ $\left.\left(\mathrm{C}_{6} \mathrm{H}_{7} \mathrm{NO}\right)_{2}\left(\mathrm{H}_{2} \mathrm{O}\right)_{4}\right]$ or $\left[\mathrm{MnCl}_{2}(3 \mathrm{MePNO})\left(\mathrm{H}_{2} \mathrm{O}\right)_{2}\right]_{2}$ (III). The $\mathrm{Mn}^{\mathrm{II}}$ atoms are found in pseudo-octahedral environments for each of the three complexes. Compound I forms a coordination polymer with alternating pairs of bridging $\mathrm{N}$-oxide and chloride ligands. The coordination environment is defined by two PNO bridging $\mathrm{O}$ atoms, two chloride bridging atoms, a terminal chloride, and a terminal water. Compound II also forms a coordination polymer with a similar metal cation; however, the coordination polymer is bridged between $\mathrm{Mn}^{\mathrm{II}}$ atoms by both a single chloride and $2 \mathrm{MePNO}$. The distorted octahedrally coordinated metal cation is defined by two bridging $2 \mathrm{MePNO}$ trans to each other, two chlorides, also trans to one another in the equatorial (polymeric) plane, and a terminal chloride and terminal water. Finally, complex III forms a dimer with two bridging 3MePNOs, two terminal chlorides and two terminal waters forming the six-coordinate metal environment. All three compounds exhibit hydrogen bonding between the coordinating water(s) and terminal chlorides.

\section{Chemical context}

The utility of aromatic $N$-oxides to facilitate organic oxotransfer reactions has been well documented over the years (see, for example, Eppenson, 2003). Many of these reactions are actually catalyzed by transition metal interactions with the $N$-oxide ligands (see, for example, Moustafa et al., 2014). Furthermore, $N$-oxide metal interactions have recently attracted much interest in a variety of other areas, including metal organic frameworks (MOFs) (Hu et al., 2014). These MOFs synthesized using $N$-oxide derivatives take advantage of the multiple binding modes of the $s p^{3} \mathrm{O}$ atom and the ease of modification of the organic backbone of the $N$-oxide. The utility of the MOFs has been examined in areas such as catalysis (Liu et al., 2014) and sensors (Hu et al., 2014). The constructs extend to the supramolecular study of coordination polymers that have been found in this type of complex 
because of their incredible versatility as ligands (Sarma \& Baruah, 2011).

In this context, we report the synthesis and solid-state structures of three pyridine $\mathrm{N}$-oxide manganese(II) complexes. Notably, we used the ligands pyridine $\mathrm{N}$-oxide, 2-methylpyridine $\mathrm{N}$-oxide, and 3-methylpyridine $\mathrm{N}$-oxide to study the impact of substitution of the pyridine on the two- and threedimensional solid-state structures. The pyridine $N$-oxide (PNO) and 2-methylpyridine $N$-oxide (2MePNO) complexes form coordination polymers with subtle differences. The 3-methylpyridine $N$-oxide (3MePNO), however, forms a dimeric complex.
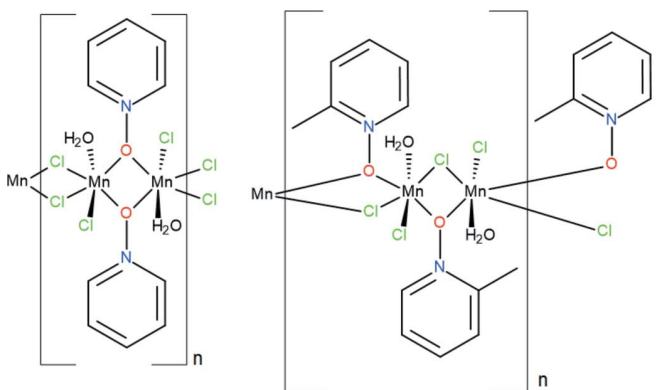

II

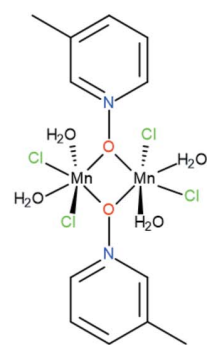

III

\section{Structural commentary}

Complex I exhibits the repeating motif of $\left[\mathrm{MnCl}_{2}-\right.$ $\left.(\mathrm{PNO})\left(\mathrm{H}_{2} \mathrm{O}\right)\right]_{n}$ and crystallizes in the triclinic space group $P \overline{1}$, containing two formula units per unit cell (Fig. 1). The coordination sphere around each $\mathrm{Mn}^{\mathrm{II}}$ atom is a distorted octahedron, with the equatorial atoms being two bridging chlorides alternating with two bridging pyridine $N$-oxide (PNO) molecules (Fig. 2). In the equatorial plane, the bridging chlorides and the bridging pyridine $\mathrm{N}$-oxides are cis to one another. The axial positions are a terminal chloride and a water molecule. The Mn1-O1 bond length is 2.177 (3) $\AA$, whereas the $\mathrm{Mn} 1-\mathrm{O} 1^{\mathrm{vii}}$ bond length is slightly longer at 2.182 (3) $\AA$ for the bridging PNO [symmetry code (vii) $-x+1$, $-y+1,-z+1]$. The bridging chlorides are found to have

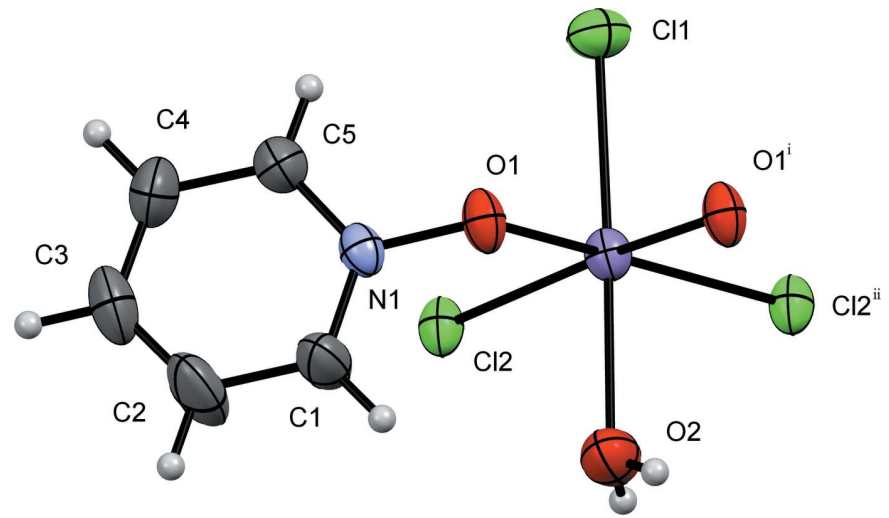

Figure 1

A view of compound I, showing the atom labeling. Displacement ellipsoids are drawn at the $50 \%$ probability level. [Symmetry codes: (i) $-x+1,-y+1,-z+1$; (ii) $-x,-y+1,-z+1]$

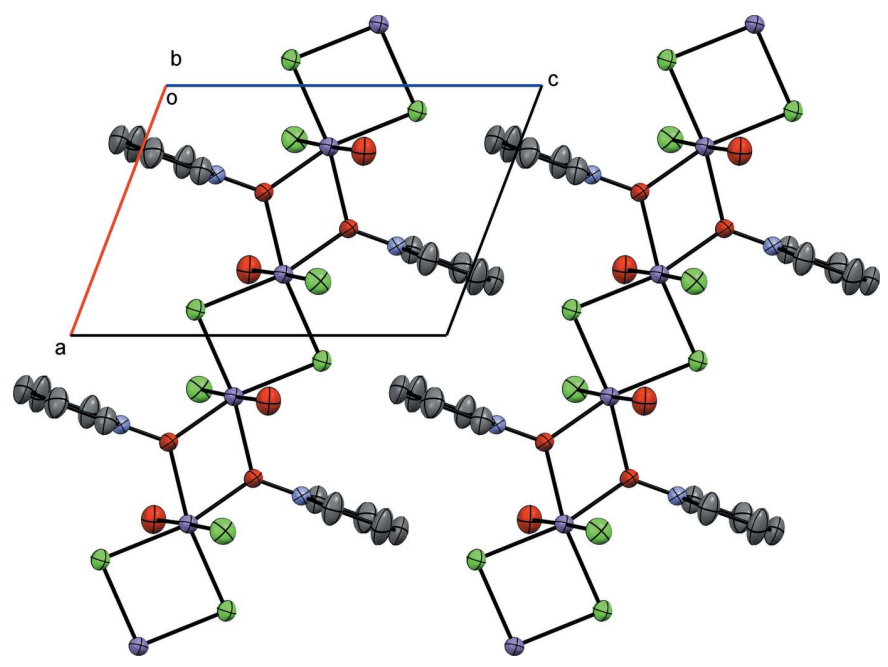

Figure 2

Crystal packing diagram of compound $\mathbf{I}$, viewed along the $b$ axis. $\mathrm{H}$ atoms have been omitted for clarity.

$\mathrm{Mn}-\mathrm{Cl} 2$ distances of 2.5240 (19) and 2.532 (19) $\AA$, respectively. Axially, the water is located 2.250 (3) $\AA$ from the $\mathrm{Mn}^{\mathrm{II}}$ cation and the terminal chloride is at 2.479 (2) $\AA$. The bond angles around the equator are severely compressed at the two bridging $\mathrm{N}$-oxides, with the $\mathrm{O} 1-\mathrm{Mn} 1-\mathrm{O} 1^{\mathrm{i}}$ angle observed at $72.03(10)^{\circ}$. The remaining three angles are found to all be similar at $95.58(7)\left(\mathrm{Cl} 2-\mathrm{Mn} 1-\mathrm{Cl} 2^{\mathrm{i}}\right), 96.80(8)(\mathrm{O} 1-\mathrm{Mn} 1-$ $\mathrm{Cl} 2)$, and $94.69(9)^{\circ}\left(\mathrm{O}^{\mathrm{vii}}-\mathrm{Mn} 1-\mathrm{Cl}^{\mathrm{vii}}\right)$. Axially, the bond angle from the water through manganese(II) and the terminal chloride $(\mathrm{O} 2-\mathrm{Mn} 1-\mathrm{Cl} 1)$ is nearly linear at $177.36(8)^{\circ}$.

Complex II, $\left[\mathrm{MnCl}_{2}(2 \mathrm{MePNO})\left(\mathrm{H}_{2} \mathrm{O}\right)\right]_{n}$, posseses a metal environment similar to complex $\mathbf{I}$ and crystallizes in the orthorhombic space group $P 2_{1} 2_{1} 2_{1}$. The major difference in structure $\mathbf{I I}$ is in the bridging network, where the chlorides and $\mathrm{N}$-oxides are trans to one another rather than cis as in I (Figs. 3 and 4). The pseudo-octahedral environment includes an $\mathrm{Mn} 1-\mathrm{Cl} 1$ bond length of $2.516(4) \AA$ and an Mn1-O1 $(N$ -

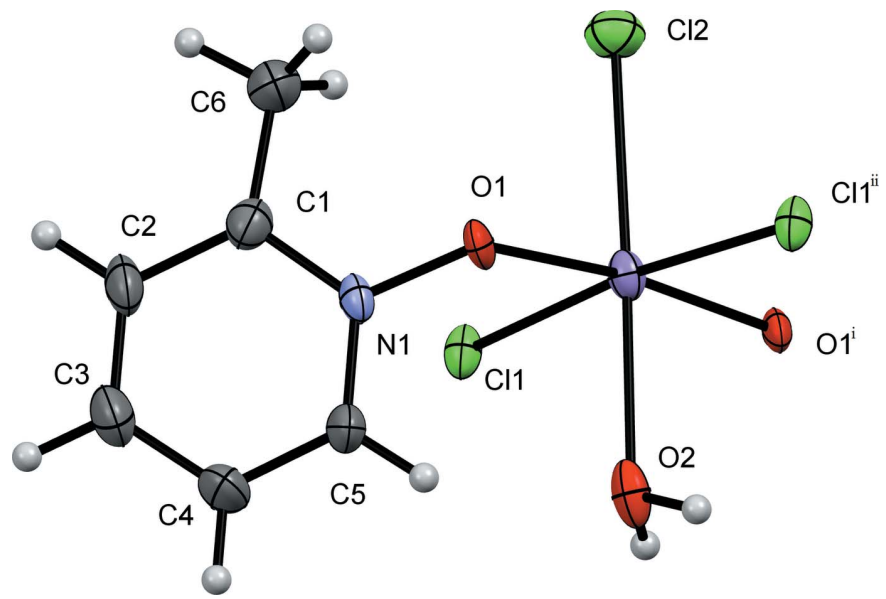

Figure 3

A view of compound II, showing the atom labeling. Displacement ellipsoids are drawn at the $50 \%$ probability level. [Symmetry codes: (i) $-x-1, y+\frac{3}{2},-z+\frac{3}{2}$; (ii) $-x, y+\frac{3}{2},-z+\frac{3}{2}$.] 
Table 1

Hydrogen-bond geometry $\left(\AA{ }^{\circ}\right)$ for $\mathbf{I}$.

\begin{tabular}{lllll}
\hline$D-\mathrm{H} \cdots A$ & $D-\mathrm{H}$ & $\mathrm{H} \cdots A$ & $D \cdots A$ & $D-\mathrm{H} \cdots A$ \\
\hline $\mathrm{O} 2-\mathrm{H} 2 A \cdots \mathrm{Cl} 1^{\mathrm{i}}$ & $0.83(2)$ & $2.53(2)$ & $3.348(4)$ & $168(4)$ \\
$\mathrm{O} 2-\mathrm{H} 2 B \cdots \mathrm{Cl} 1^{\mathrm{ii}}$ & $0.82(2)$ & $2.52(3)$ & $3.232(4)$ & $147(4)$ \\
\hline
\end{tabular}

Symmetry codes: (i) $-x,-y+1,-z+1$; (ii) $x, y-1, z$.

oxide) bond length of 2.170 (6) $\AA$, with a Cl1-Mn1-O1 bond angle of $84.37(19)^{\circ}$. The bond angle across the $\mathrm{Cl}$ atoms, $\mathrm{Cl} 1-\mathrm{Mn} 1-\mathrm{Cl}^{\mathrm{viii}}$, is $174.02(5)^{\circ}$ and across the $\mathrm{O}$ atoms of 2MePNO, O1-Mn1-O $1^{\text {ix }}$, is $173.12(6)^{\circ}$; a slight compression is observed across the bridges [symmetry codes: (viii) $-x-1, y+\frac{3}{2},-z+\frac{3}{2}$; (ix) $\left.-x, y+\frac{3}{2},-z+\frac{3}{2}\right]$. The axial (nonbridging) $\mathrm{Mn} 1-\mathrm{Cl} 2$ bond length is 2.503 (4) $\AA$, while the axial water is found at a distance of 2.268 (6) $\AA$ from the metal center.

The dimeric complex III, $\left[\mathrm{MnCl}_{2}(3 \mathrm{MePNO})\left(\mathrm{OH}_{2}\right)_{2}\right]_{2}$, crystallizes in the triclinic $P \overline{1}$ space group, with the inversion center sitting in the center of the dimer (Fig. 5). The 3-methyl derivative does not form a coordination polymer but discrete dimeric molecules. The structure possesses two bridging 3MePNO ligands, four terminal chlorides, and four terminal waters. Two waters and two chlorides are in the equatorial plane coincident with the $N$-oxide bridge, and the other equivalents are axial in the pseudo-octahedral geometry around the $\mathrm{Mn}^{\mathrm{II}}$ atoms. The $\mathrm{Mn} 1-\mathrm{Cl} 1$ and $\mathrm{Mn} 1-\mathrm{Cl} 2$ bond lengths are 2.4601 (5) and 2.4903 (19) $\AA$, respectively, with a $\mathrm{Cl} 1-\mathrm{Mn} 1-\mathrm{Cl} 2$ bond angle of $98.32(4)^{\circ}$. The bridging $N$-oxide is at a distance of 2.1791 (18) $\AA$ from Mn1-O1, with an $\mathrm{O} 1-\mathrm{Mn} 1-\mathrm{O} 1^{\mathrm{vii}}$ bond angle of $71.86(7)^{\circ}$ [symmetry code: (vii) $-x+1,-y+1,-z+1]$. The Mn1-O2(water) and Mn1O3(water) bond lengths are 2.245 (2) and 2.1696 (17) $\AA$, respectively, with an $\mathrm{O} 2-\mathrm{Mn} 1-\mathrm{O} 3$ bond angle of $85.83(7)^{\circ}$.

The formation of the polymeric structure in I and II versus the dimer in III is likely due to the steric influence of the methyl group in the 3-position in $3 \mathrm{MePNO}$ and the core constituents. One can define the $\mathrm{Mn}_{2}$ ' $N$-oxide diamond core' in each of the structures as follows: $\mathbf{I}$ is alternating $\mathrm{Mn}_{2} \mathrm{Cl}_{2}$ and $\mathrm{Mn}_{2} \mathrm{O}_{2}$ (oxygen bridges via $\mathrm{PNO}$ ) cores, $\mathbf{I I}$ is $\mathrm{Mn}_{2} \mathrm{ClO}$ (oxygen bridge via $2 \mathrm{MePNO}$ ) and III $\mathrm{Mn}_{2} \mathrm{O}_{2}$ (oxygen bridges via $3 \mathrm{MePNO})$. In $\mathbf{I}$, the unsubstituted pyridine $N$-oxide group

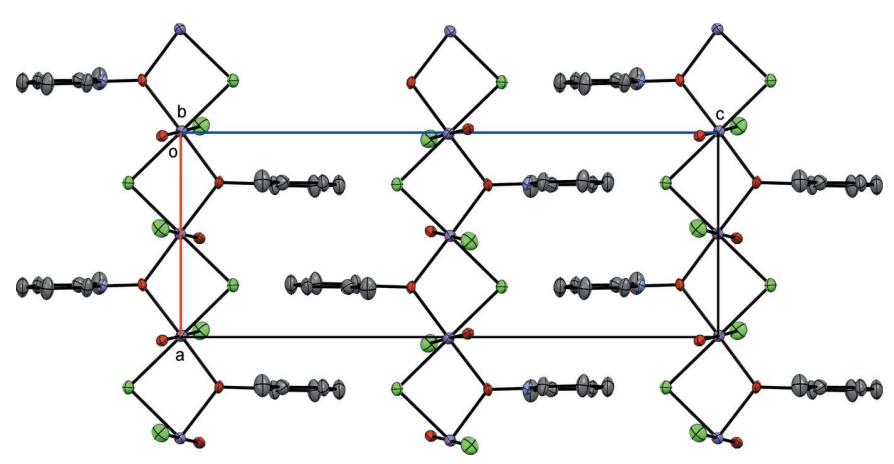

Figure 4

Crystal packing diagram of compound II, viewed along the $b$ axis. $\mathrm{H}$ atoms have been omitted for clarity.
Table 2

Hydrogen-bond geometry $\left(\AA,^{\circ}\right)$ for II.

\begin{tabular}{lllll}
\hline$D-\mathrm{H} \cdots A$ & $D-\mathrm{H}$ & $\mathrm{H} \cdots A$ & $D \cdots A$ & $D-\mathrm{H} \cdots A$ \\
\hline $\mathrm{O} 2-\mathrm{H} 2 A \cdots \mathrm{Cl} 2^{\mathrm{iii}}$ & 0.90 & 2.49 & $3.205(7)$ & 137 \\
$\mathrm{O} 2-\mathrm{H} 2 B \cdots \mathrm{Cl}^{\mathrm{iv}}$ & 0.89 & 2.26 & $3.145(7)$ & 169 \\
\hline
\end{tabular}

Symmetry codes: (iii) $x, y+1, z$; (iv) $x-\frac{1}{2},-y+\frac{3}{2},-z+1$.

does not generate as much steric strain, allowing for polymer formation. In II, the core is formed to permit alternating up and down pyridine $N$-oxides with the 2-methyl substituents also facing in opposite directions. This limits the steric interactions and the $N$-oxide slightly tilts out of the polymeric core line to allow the methyl group to effect less steric interactions. In III, the methyl group appears to inhibit polymer formation due to the position of this bulky substituent. Subsequently, when the polymer is not formed, an extra water molecule is required to fill the sixth coordination site on the metal cation occupied by a bridging atom in I and II.

\section{Supramolecular features}

The packing of $\mathbf{I}$ forms a coordination polymer of alternating bis-bridges of two chlorides and two pyridine $\mathrm{N}$-oxides in the $a$-axis direction (Fig. 2). The aromatic rings stack at 6.860 (7) $\AA$, outside of $\pi$-stacking distance due to the alternating chloride and pyridine $N$-oxide bridges. The single water molecule is locked into weak hydrogen-bonding interactions in two different modes. One hydrogen-bond interaction $(\mathrm{H} 2 A)$ is located down the bridge to the terminal chloride (Cl1), on the adjacent $\mathrm{Mn}^{\mathrm{II}}$ atom, and the $\mathrm{O} 2-\mathrm{H} 2 A \cdots \mathrm{Cl} 1^{\mathrm{i}}$ distance is 2.53 (2) $\AA$. The other hydrogen-bond interaction $(\mathrm{H} 2 B)$ is across to the next polymeric chain with $\mathrm{Cl} 1$; the $\mathrm{O} 2-$ $\mathrm{H} 2 B \cdots \mathrm{Cl} 1^{\mathrm{ii}}$ distance is 2.52 (3) $\AA$ (see Table 1 for hydrogenbond details and symmetry codes).

Complex II packs as a coordination polymer in the $a$ direction similar to I (Fig. 4). However, as I has alternating pyridine $N$-oxide and chloride bridges (placing these ligands cis to one another), II has a single 2-methylpyridine $N$-oxide

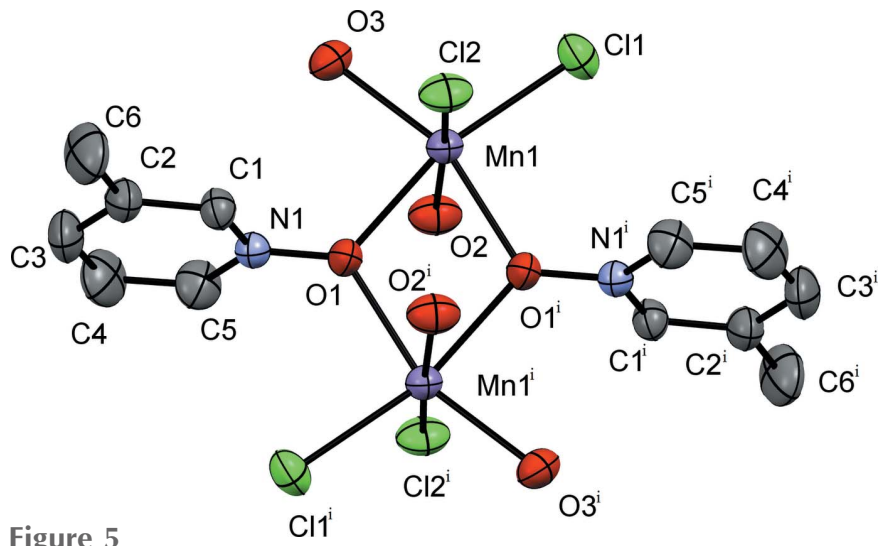

Figure 5

A view of the molecular structure of compound III, showing the atom labeling. Displacement ellipsoids are drawn at the $50 \%$ probability level. $\mathrm{H}$ atoms have been omitted for clarity. [Symmetry code: (i) $-x-1,-y+1$, $-z+1$.] 
Table 3

Hydrogen-bond geometry $\left(\AA,^{\circ}\right)$ for III.

\begin{tabular}{lllll}
\hline$D-\mathrm{H} \cdots A$ & $D-\mathrm{H}$ & $\mathrm{H} \cdots A$ & $D \cdots A$ & $D-\mathrm{H} \cdots A$ \\
\hline $\mathrm{O} 2-\mathrm{H} 2 B \cdots \mathrm{Cl} 1^{\mathrm{v}}$ & $0.80(2)$ & $2.38(2)$ & $3.147(3)$ & $161(2)$ \\
$\mathrm{O} 3-\mathrm{H} 3 A \cdots \mathrm{Cl} 2^{\mathrm{vi}}$ & $0.86(2)$ & $2.28(2)$ & $3.120(2)$ & $167(2)$ \\
\hline
\end{tabular}

Symmetry codes: (v) $-x,-y+1,-z+1 ;$ (vi) $-x,-y,-z+1$.

and a single chloride in each bridge. Similar to I, the hydrogenbonding interactions are to a terminal chloride $(\mathrm{Cl} 2)$ on the adjacent $\mathrm{Mn}^{\mathrm{II}}$ atom. There are two observed interactions, viz. $\mathrm{O} 2-\mathrm{H} 2 A \cdots \mathrm{Cl} 2^{\mathrm{iii}}$ with a distance of $2.49 \AA$ and $\mathrm{O} 2-$ $\mathrm{H} 2 B \cdots \mathrm{Cl}^{\mathrm{iv}}$ with a distance of $2.26 \AA$ (see Table 2 for hydrogen-bond details and symmetry codes). The $\mathrm{H} 2 A \cdots \mathrm{Cl} 2$ interaction is in the coordination polymer and the $\mathrm{H} 2 B \cdots \mathrm{Cl} 2$ interaction is across the polymeric chains. Similar to $\mathbf{I}$, the aromatic rings stack too far apart to be interacting in the $a$ direction, at a distance of 6.862 (11) $\mathrm{\text {. }}$.

As noted above, compound III does not form a coordination polymer but is observed in the solid state as a dimer with two water molecules for each $\mathrm{Mn}^{\mathrm{II}}$ atom (versus one aqua equivalent in I and II) (Fig. 5). The aromatic inter-centroid distance is longer than in the other two molecules, at 7.902 (7) $\mathrm{A}$. In compound III, a single water molecule hydrogen bonds from the equatorial plane of one dimer to an axial chloride on another dimer. Conversely, the axial water hydrogen bonds to an equatorial chloride on a different dimer. These interactions are found to be $\mathrm{O} 2-\mathrm{H} 2 B \cdots \mathrm{Cl}^{\mathrm{v}}$ [distance $2.38(2) \AA$ ] and $\mathrm{O} 3-\mathrm{H} 3 A \cdots \mathrm{Cl} 2^{\mathrm{vi}}$ [distance 2.28 (2) $\AA$ ] (see Table 3 for hydrogen-bond details and symmetry codes).

\section{Database survey}

A search in the Cambridge Structural Database (CSD; Groom et al., 2016) for aromatic $N$-oxides bound to manganese returned 87 entries. Similar $N$-oxides with simple counter-ions in the list include 4,4'-dipyridine $N, N^{\prime}$-dioxide [FIVHAU (Ma et al., 2005) and XOHQUH (Jiu et al., 2008)], 1,2-bis(4pyridyl)ethane $N, N^{\prime}$-dioxide (TOJDAY and TOJDIG; Sun et al., 2008), and 1,3-bis(4-pyridyl)propane $N, N^{\prime}$-dioxide (Zhang et al., 2003). Similarly, two derivatives of 3,5-dimethylpyridine $\mathrm{N}$-oxide are found in the CSD (GIWQAF and GIWQEJ; Shi et al., 2007)

\section{Synthesis and crystallization}

The title compounds were all synthesized in a similar manner. $0.200 \mathrm{~g}$ of $\mathrm{MnCl}_{2} \cdot 4 \mathrm{H}_{2} \mathrm{O}(1.01 \mathrm{mmol})$ was dissolved in a

Table 4

Experimental details.

I

Crystal data

Chemical formula

$M_{\mathrm{r}}$

Crystal system, space group

Temperature (K)

$a, b, c(\AA)$

$\alpha, \beta, \gamma\left({ }^{\circ}\right)$

$V\left(\AA^{3}\right)$

$Z$

Radiation type

$\mu\left(\mathrm{mm}^{-1}\right)$

Crystal size $(\mathrm{mm})$

Data collection

Diffractometer

Absorption correction

$T_{\min }, T_{\max }$

No. of measured, independent and observed $[I>2 \sigma(I)]$ reflections

$R_{\text {int }}$

$(\sin \theta / \lambda)_{\max }\left(\AA^{-1}\right)$

Refinemen

$R\left[F^{2}>2 \sigma\left(F^{2}\right)\right], w R\left(F^{2}\right), S$

No. of reflections

No. of parameters

No. of restraints

$\mathrm{H}$-atom treatment

$\Delta \rho_{\max }, \Delta \rho_{\min }\left(\mathrm{e} \AA^{-3}\right)$

Absolute structure

Absolute structure parameter

$\left[\mathrm{MnCl}_{2}\left(\mathrm{C}_{5} \mathrm{H}_{5} \mathrm{NO}\right)\left(\mathrm{H}_{2} \mathrm{O}\right)\right]$
238.96
Triclinic, $P \overline{1}$
173
$6.897(2), 7.050(1), 9.853(3)$
$101.042(7), 109.559(10)$,
$\quad 94.196(6)$
$438.2(2)$
2
$\mathrm{Mo} \mathrm{K \alpha}$
2.06
$0.29 \times 0.18 \times 0.13$

Rigaku XtalLab mini CCD Multi-scan (REQAB; Rigaku, 1998)

$0.613,0.765$

4655, 2004, 1770

0.040

0.651

$0.031,0.080,1.14$
2004
108
2
$\mathrm{H}$ atoms treated by a mixture of
$\quad$ independent and constrained
$\quad$ refinement
$0.40,-0.44$
-

\section{II}

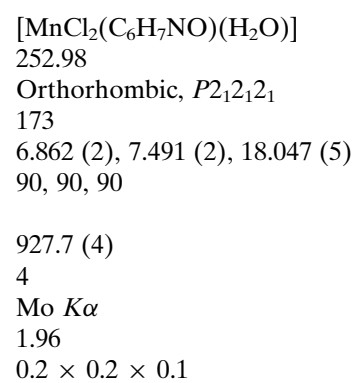

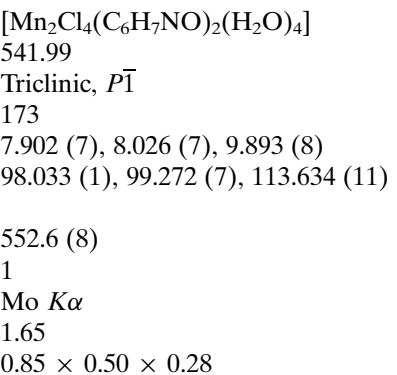

Rigaku XtalLab mini CCD Multi-scan (REQAB; Rigaku, 1998)

$0.563,0.737$

$8438,2109,1800$

0.051

0.649

Rigaku XtalLab mini CCD

Multi-scan (REQAB; Rigaku, 1998)

$0.482,0.630$

$5837,2553,2375$

0.072

0.652

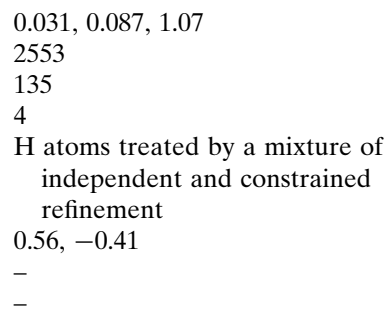

Computer programs: CrystalClearSM Expert (Rigaku, 2011), SHELXT (Sheldrick, 2015a), SHELXL2017 (Sheldrick, 2015b) and OLEX2 (Dolomanov et al., 2009). 
minimal amount of methanol, approximately $10 \mathrm{ml}$. Two stoichiometric equivalents of the appropriate $\mathrm{N}$-oxide were also dissolved in approximately $20 \mathrm{ml}$ of methanol (PNO: 0.191 g, 2.02 mmol; 2MePNO: 0.220 g, $2.02 \mathrm{mmol}$; 3MePNO: $0.220 \mathrm{~g}, 2.02 \mathrm{mmol})$. The solutions were stirred for approximately $10 \mathrm{~min}$; during each reaction, a brown solution was observed upon mixing. The reaction solution was then allowed to sit and brown crystals were grown by slow evaporation in the near quantitative yields reported below based on the manganese(II) chloride starting material. The FT-IR spectra of the complexes all exhibit broad absorbances in the 3400$3000 \mathrm{~cm}^{-1}$ region due to the $v(\mathrm{O}-\mathrm{H})$ of the coordinating water molecules, as well as the characteristic $v(\mathrm{~N}-\mathrm{O})$ of the $N$-oxide pyridyl moiety in the $1250-1150 \mathrm{~cm}^{-1}$ region noted previously (Mautner et al., 2017).

Compound I, $\mathrm{Mn}(\mathrm{PNO}) \mathrm{Cl}_{2} \cdot \mathrm{H}_{2} \mathrm{O}$, yield $0.215 \mathrm{~g}(90.3 \%)$. Selected IR bands (ATR, FT-IR, KBr composite, $\mathrm{cm}^{-1}$ ): 3364 ( $m, b r), 3235(m, b r), 3068(m, b r), 1660(w), 1471(w), 1214$ $(\mathrm{m}), 1205(\mathrm{~m}), 1023(\mathrm{~s}), 831(\mathrm{~s}), 780(\mathrm{~s}), 674(\mathrm{~s}), 556(\mathrm{~s})$. Elemental analysis for $\mathrm{MnCl}_{2} \mathrm{C}_{5} \mathrm{H}_{7} \mathrm{NO}_{2}$, calculated (\%): C 25.13, H 2.95, N 5.86; found (\%): C 25.22, H 2.96, N 5.87.

Compound II, $\mathrm{Mn}(2 \mathrm{MePNO}) \mathrm{Cl}_{2} \cdot \mathrm{H}_{2} \mathrm{O}$, yield $0.227 \mathrm{~g}$ (87.9\%). Selected IR bands (ATR, FT-IR, KBr composite, $\left.\mathrm{cm}^{-1}\right)$ : $3410(m, b r), 3247(m, b r), 3073(m, b r), 1716(w), 1619$ $(w), 1578(w), 1421(m), 1264(m), 1154(m), 1029(s), 831(s)$, $799(s), 684(s), 584(s)$. Elemental analysis for $\mathrm{MnCl}_{2^{-}}$ $\mathrm{C}_{6} \mathrm{H}_{9} \mathrm{NO}_{2}$, calculated (\%): C 28.48, H 3.59, N 5.53; found (\%): C 28.75, H 3.53, N 5.28.

Compound III, $\mathrm{Mn}_{2}(3 \mathrm{MePNO})_{2} \mathrm{Cl}_{4} \cdot 4 \mathrm{H}_{2} \mathrm{O}$, yield $0.231 \mathrm{~g}$ $(89.5 \%)$. Selected IR bands (ATR, FT-IR, KBr composite, $\left.\mathrm{cm}^{-1}\right)$ : $3374(m, b r), 3251$ ( $\left.m, b r\right), 3094(m, b r), 1663(w)$, 1614(w), $1492(m), 1261(m), 1164(m), 1019(s), 946(s), 750$ $(s), 672(s)$. Elemental analysis for $\mathrm{Mn}_{2} \mathrm{Cl}_{4} \mathrm{C}_{12} \mathrm{H}_{22} \mathrm{~N}_{2} \mathrm{O}_{6}$, calculated (\%): C 26.59, H 4.09, N 5.16; found (\%): C 26.53, H 4.04, N 5.21.

\section{Refinement}

Crystal data, data collection and structure refinement details are summarized in Table 4. All carbon-bound $\mathrm{H}$ atoms were positioned geometrically and refined as riding, with $\mathrm{C}-\mathrm{H}=$ 0.95 or $0.98 \AA$ and $U_{\text {iso }}(\mathrm{H})=1.2 U_{\text {eq }}(\mathrm{C})$ or $U_{\text {iso }}(\mathrm{H})=1.5 U_{\text {eq }}(\mathrm{C})$ for $\mathrm{C}(\mathrm{H})$ and $\mathrm{CH}_{3}$ groups, respectively. In order to ensure chemically meaningful $\mathrm{O}-\mathrm{H}$ distances for the bound water molecules in compound $\mathbf{I}$, the $\mathrm{H} 2 A-\mathrm{O} 2$ and $\mathrm{H} 2 B-\mathrm{O} 2$ distances were restrained to a target value of 0.84 (2) $\AA$ (using a DFIX command in SHELXL2017; Sheldrick, 2015b). In compound II, water $\mathrm{H}$ atoms were refined as riding, with the $\mathrm{O}-\mathrm{H}$ distance constrained to $0.892 \AA$ and $U_{\text {iso }}(\mathrm{H})=$ $1.5 U_{\text {eq }}(\mathrm{O})$ using an AFIX 7 command, and in compound III, $\mathrm{H} 2 A-\mathrm{O} 2, \mathrm{H} 2 B-\mathrm{O} 2, \mathrm{H} 3 A-\mathrm{O} 3$, and $\mathrm{H} 3 B-\mathrm{O} 3$ were restrained using DFIX as for compound I. A rotating-group model was applied for the methyl groups. Structure refinement of II exhibits inversion twinning. Several crystals were tried and the centrosymmetric space group Pnma was tested. In all cases, there was a significant reduction in the $R$ value for the inversion twinning $P 2_{1} 2_{1} 2_{1}$ solution.

\section{Acknowledgements}

The authors would like to thank Armstrong State University, Department of Chemistry and Physics, for financial support of this work.

\section{References}

Dolomanov, O. V., Bourhis, L. J., Gildea, R. J., Howard, J. A. K. \& Puschmann, H. (2009). J. Appl. Cryst. 42, 339-341.

Eppenson, J. H. (2003). Adv. Inorg. Chem. 54, 157-202.

Groom, C. R., Bruno, I. J., Lightfoot, M. P. \& Ward, S. C. (2016). Acta Cryst. B72, 171-179.

Hu, Z. C., Deibert, B. J. \& Li, J. (2014). Chem. Soc. Rev. 43, 58155840 .

Jiu, J., Blake, A. J., Champness, N. R., Hubberstey, P., Wilson, C. \& Schröder, M. (2008). Inorg. Chem. 47, 8652-8664.

Liu, J., Chen, L., Cui, H., Zhang, Y., Zhang, L. \& Su, C.-Y. (2014). Chem. Soc. Rev. 43, 6011-6061.

Ma, B.-Q., Sun, H.-L. \& Gao, S. (2005). Inorg. Chem. 44, 837-839.

Mautner, F. A., Berger, C., Fischer, R. C., Massoud, S. S. \& Vicente, R. (2017). Polyhedron, 134, 126-134.

Moustafa, M. E., Boyle, P. D. \& Puddephatt, R. J. (2014). Organometallics, 33, 5402-5413.

Rigaku (1998). REQAB. Rigaku Corporation, Tokyo, Japan.

Rigaku (2011). CrystalClearSM Expert. Rigaku Corporation, Tokyo, Japan.

Sarma, R. \& Baruah, J. B. (2011). Solid State Sci. 13, 1692-1700.

Sheldrick, G. M. (2015a). Acta Cryst. A71, 3-8.

Sheldrick, G. M. (2015b). Acta Cryst. C71, 3-8.

Shi, J.-M., Liu, Z., Li, W.-N., Zhao, H. Y. \& Liu, L.-D. (2007). J. Coord. Chem. 60, 1077-1082.

Sun, H.-L., Wang, Z.-M., Gao, S. \& Batten, S. R. (2008). CrystEngComm, 10, 1796-1802.

Zhang, L.-P., Lu, W.-J. \& Mak, T. C. (2003). Chem. Commun. 22, 2830-2831. 


\section{supporting information}

Acta Cryst. (2017). E73, 1434-1438 [https://doi.org/10.1107/S2056989017012038]

\section{Manganese(II) chloride complexes with pyridine N-oxide (PNO) derivatives and their solid-state structures}

\section{Linda Kang, Genevieve Lynch, Will Lynch and Clifford Padgett}

Computing details

For all structures, data collection: CrystalClearSM Expert (Rigaku, 2011); cell refinement: CrystalClearSM Expert (Rigaku, 2011); data reduction: CrystalClearSM Expert (Rigaku, 2011); program(s) used to solve structure: SHELXT (Sheldrick, 2015a); program(s) used to refine structure: SHELXL2017 (Sheldrick, 2015b); molecular graphics: OLEX2 (Dolomanov et al., 2009); software used to prepare material for publication: OLEX2 (Dolomanov et al., 2009).

catena-poly[[aquachloridomanganese(II)]-di- $\mu$-chlorido-[aquachloridomanganese(II)]-bis( $\mu$-pyridine $N$-oxide)]

(I)

\section{Crystal data}

$\left[\mathrm{MnCl}_{2}\left(\mathrm{C}_{5} \mathrm{H}_{5} \mathrm{NO}\right)\left(\mathrm{H}_{2} \mathrm{O}\right)\right]$

$M_{r}=238.96$

Triclinic, $P \overline{1}$

$a=6.897(2) \AA$

$b=7.050(1) \AA$

$c=9.853(3) \AA$

$\alpha=101.042(7)^{\circ}$

$\beta=109.559(10)^{\circ}$

$\gamma=94.196(6)^{\circ}$

$V=438.2(2) \AA^{3}$

Data collection

Rigaku XtalLab mini CCD diffractometer

$\omega$ scans

Absorption correction: multi-scan

(REQAB; Rigaku, 1998)

$T_{\min }=0.613, T_{\max }=0.765$

4655 measured reflections

\section{Refinement}

Refinement on $F^{2}$

Least-squares matrix: full

$R\left[F^{2}>2 \sigma\left(F^{2}\right)\right]=0.031$

$w R\left(F^{2}\right)=0.080$

$S=1.14$

2004 reflections

108 parameters

2 restraints

Primary atom site location: dual
$Z=2$

$F(000)=238$

$D_{\mathrm{x}}=1.811 \mathrm{Mg} \mathrm{m}^{-3}$

Mo $K \alpha$ radiation, $\lambda=0.71073 \AA$

Cell parameters from 1189 reflections

$\theta=2.3-27.5^{\circ}$

$\mu=2.06 \mathrm{~mm}^{-1}$

$T=173 \mathrm{~K}$

Prism, clear brown

$0.29 \times 0.18 \times 0.13 \mathrm{~mm}$

2004 independent reflections

1770 reflections with $I>2 \sigma(I)$

$R_{\text {int }}=0.040$

$\theta_{\max }=27.6^{\circ}, \theta_{\min }=2.3^{\circ}$

$h=-8 \rightarrow 8$

$k=-9 \rightarrow 9$

$l=-12 \rightarrow 12$

Hydrogen site location: mixed

$\mathrm{H}$ atoms treated by a mixture of independent and constrained refinement

$w=1 /\left[\sigma^{2}\left(F_{0}^{2}\right)+0.444 P\right]$

where $P=\left(F_{\mathrm{o}}^{2}+2 F_{\mathrm{c}}{ }^{2}\right) / 3$

$(\Delta / \sigma)_{\max }=0.001$

$\Delta \rho_{\max }=0.40 \mathrm{e} \AA^{-3}$

$\Delta \rho_{\min }=-0.44$ e $\AA^{-3}$ 


\section{Special details}

Geometry. All esds (except the esd in the dihedral angle between two 1.s. planes) are estimated using the full covariance matrix. The cell esds are taken into account individually in the estimation of esds in distances, angles and torsion angles; correlations between esds in cell parameters are only used when they are defined by crystal symmetry. An approximate (isotropic) treatment of cell esds is used for estimating esds involving l.s. planes.

Fractional atomic coordinates and isotropic or equivalent isotropic displacement parameters $\left(\AA^{2}\right)$

\begin{tabular}{lllll}
\hline & $x$ & $y$ & $z$ & $U_{\text {iso }} * / U_{\text {eq }}$ \\
\hline Mn1 & $0.24478(6)$ & $0.51944(6)$ & $0.49588(4)$ & $0.02748(12)$ \\
C1 & $0.21608(12)$ & $0.81797(10)$ & $0.39668(9)$ & $0.04150(19)$ \\
O1 & $0.5727(3)$ & $0.6094(3)$ & $0.63106(19)$ & $0.0327(4)$ \\
N1 & $0.6425(3)$ & $0.6894(3)$ & $0.7767(2)$ & $0.0283(5)$ \\
C1 & $0.6940(6)$ & $0.5762(5)$ & $0.8731(3)$ & $0.0493(8)$ \\
H1 & 0.681940 & 0.441595 & 0.840110 & $0.059^{*}$ \\
C12 & $0.10246(10)$ & $0.65268(10)$ & $0.69397(7)$ & $0.03317(16)$ \\
O2 & $0.2634(4)$ & $0.2541(3)$ & $0.5927(3)$ & $0.0447(5)$ \\
H2A & $0.146(4)$ & $0.218(6)$ & $0.594(5)$ & $0.081(16)^{*}$ \\
H2B & $0.305(7)$ & $0.162(5)$ & $0.551(5)$ & $0.087(16)^{*}$ \\
C2 & $0.7655(7)$ & $0.6608(6)$ & $1.0222(4)$ & $0.0655(11)$ \\
H2 & 0.802254 & 0.582800 & 1.090313 & $0.079^{*}$ \\
C3 & $0.7826(6)$ & $0.8564(6)$ & $1.0704(3)$ & $0.0543(9)$ \\
H3 & 0.829678 & 0.913307 & 1.171227 & $0.065^{*}$ \\
C4 & $0.7299(6)$ & $0.9692(5)$ & $0.9693(4)$ & $0.0532(9)$ \\
H4 & 0.742760 & 1.104037 & 1.001068 & $0.064^{*}$ \\
C5 & $0.6577(5)$ & $0.8836(5)$ & $0.8203(3)$ & $0.0421(7)$ \\
H5 & 0.619974 & 0.959566 & 0.750808 & $0.051^{*}$ \\
& & & &
\end{tabular}

Atomic displacement parameters $\left(\AA^{2}\right)$

\begin{tabular}{lllllll}
\hline & $U^{11}$ & $U^{22}$ & $U^{33}$ & $U^{12}$ & $U^{13}$ & $U^{23}$ \\
\hline Mn1 & $0.0228(2)$ & $0.0328(2)$ & $0.0259(2)$ & $0.00479(16)$ & $0.00858(16)$ & $0.00467(16)$ \\
C11 & $0.0478(4)$ & $0.0329(4)$ & $0.0481(4)$ & $0.0073(3)$ & $0.0188(4)$ & $0.0156(3)$ \\
O1 & $0.0230(9)$ & $0.0460(12)$ & $0.0238(9)$ & $0.0052(8)$ & $0.0062(8)$ & $-0.0006(8)$ \\
N1 & $0.0232(11)$ & $0.0371(12)$ & $0.0233(10)$ & $0.0033(9)$ & $0.0078(9)$ & $0.0052(9)$ \\
C1 & $0.076(2)$ & $0.0386(17)$ & $0.0325(15)$ & $0.0152(16)$ & $0.0152(16)$ & $0.0124(13)$ \\
C12 & $0.0307(3)$ & $0.0377(4)$ & $0.0267(3)$ & $0.0016(3)$ & $0.0102(3)$ & $-0.0016(3)$ \\
O2 & $0.0553(16)$ & $0.0394(13)$ & $0.0420(13)$ & $0.0117(11)$ & $0.0186(12)$ & $0.0115(10)$ \\
C2 & $0.094(3)$ & $0.073(3)$ & $0.0290(16)$ & $0.031(2)$ & $0.0135(19)$ & $0.0204(17)$ \\
C3 & $0.057(2)$ & $0.071(3)$ & $0.0249(15)$ & $0.0074(18)$ & $0.0091(15)$ & $-0.0009(15)$ \\
C4 & $0.067(2)$ & $0.0425(19)$ & $0.0391(17)$ & $-0.0022(17)$ & $0.0151(17)$ & $-0.0064(15)$ \\
C5 & $0.054(2)$ & $0.0388(17)$ & $0.0325(15)$ & $0.0027(14)$ & $0.0149(14)$ & $0.0075(13)$ \\
& & & & & &
\end{tabular}

Geometric parameters $\left(\AA,{ }^{o}\right)$

\begin{tabular}{llll}
\hline $\mathrm{Mn} 1-\mathrm{C} 11$ & $2.479(2)$ & $\mathrm{C} 1-\mathrm{C} 2$ & $1.376(5)$ \\
$\mathrm{Mn} 1-\mathrm{O} 1$ & $2.177(3)$ & $\mathrm{O} 2-\mathrm{H} 2 \mathrm{~A}$ & $0.833(19)$ \\
$\mathrm{Mn} 1-\mathrm{O} 1^{\mathrm{i}}$ & $2.182(2)$ & $\mathrm{O} 2-\mathrm{H} 2 \mathrm{~B}$ & $0.819(19)$
\end{tabular}




$$
\begin{aligned}
& \mathrm{Mn} 1-\mathrm{Cl} 2^{\mathrm{ii}} \\
& \mathrm{Mn} 1-\mathrm{Cl} 2 \\
& \mathrm{Mn} 1-\mathrm{O} 2 \\
& \mathrm{O} 1-\mathrm{N} 1 \\
& \text { N1-C1 } \\
& \text { N1-C5 } \\
& \text { C1-H1 } \\
& \mathrm{C} 11-\mathrm{Mn} 1-\mathrm{Cl} 2 \\
& \mathrm{C} 11-\mathrm{Mn} 1-\mathrm{Cl} 2^{\mathrm{ii}} \\
& \mathrm{O} 1 \text { - }-\mathrm{Mn} 1-\mathrm{Cl} \\
& \mathrm{O} 1-\mathrm{Mn} 1-\mathrm{Cl} 1 \\
& \mathrm{O} 1-\mathrm{Mn} 1-\mathrm{O} 1^{\mathrm{i}} \\
& \mathrm{O} 1-\mathrm{Mn} 1-\mathrm{Cl} 2^{\mathrm{ii}} \\
& \mathrm{O} 1^{\mathrm{i}}-\mathrm{Mn} 1-\mathrm{Cl} 2^{\mathrm{ii}} \\
& \mathrm{O} 1-\mathrm{Mn} 1-\mathrm{Cl} 2 \\
& \mathrm{O} 1-\mathrm{Mn} 1-\mathrm{Cl} 2 \\
& \mathrm{O} 1-\mathrm{Mn} 1-\mathrm{O} 2 \\
& \mathrm{O} 1-\mathrm{Mn} 1-\mathrm{O} 2 \\
& \mathrm{Cl} 2-\mathrm{Mn} 1-\mathrm{Cl}^{\mathrm{ii}} \\
& \mathrm{O} 2-\mathrm{Mn} 1-\mathrm{Cl} 1 \\
& \mathrm{O} 2-\mathrm{Mn} 1-\mathrm{Cl} 2^{\mathrm{ii}} \\
& \text { N1-O1-Mn1 } \\
& \mathrm{N} 1-\mathrm{O} 1-\mathrm{Mn} 1 \\
& \mathrm{C} 1-\mathrm{N} 1-\mathrm{C} 5
\end{aligned}
$$$$
2.5324(19)
$$$$
2.5240(19)
$$$$
2.250(3)
$$$$
1.341(3)
$$$$
1.331(4)
$$$$
1.339(4)
$$$$
0.9300
$$$$
93.43(6)
$$$$
92.57(6)
$$$$
95.12(8)
$$$$
93.65(7)
$$$$
72.02(10)
$$$$
165.77 \text { (6) }
$$$$
94.69(9)
$$$$
166.32(5)
$$$$
96.81(8)
$$$$
86.96(9)
$$$$
87.46(10)
$$$$
95.58(7)
$$$$
177.42(7)
$$$$
87.40(8)
$$$$
84.01(9)
$$$$
107.98(10)
$$$$
123.78(14)
$$$$
126.50 \text { (16) }
$$$$
119.9 \text { (2) }
$$

$\begin{array}{ll}\mathrm{C} 2-\mathrm{H} 2 & 0.9300 \\ \mathrm{C} 2-\mathrm{C} 3 & 1.353(5) \\ \mathrm{C} 3-\mathrm{H} 3 & 0.9300 \\ \mathrm{C} 3-\mathrm{C} 4 & 1.364(5) \\ \mathrm{C} 4-\mathrm{H} 4 & 0.9300 \\ \mathrm{C} 4-\mathrm{C} 5 & 1.377(4) \\ \mathrm{C} 5-\mathrm{H} 5 & 0.9300 \\ & \\ \mathrm{C} 5-\mathrm{N} 1-\mathrm{O} 1 & 118.0(2) \\ \mathrm{N} 1-\mathrm{C} 1-\mathrm{H} 1 & 120.4 \\ \mathrm{~N} 1-\mathrm{C} 1-\mathrm{C} 2 & 119.1(3) \\ \mathrm{C} 2-\mathrm{C} 1-\mathrm{H} 1 & 120.4 \\ \mathrm{Mn} 1-\mathrm{C} 12-\mathrm{Mn} 1 & 84.42(7) \\ \mathrm{Mn} 1-\mathrm{O} 2-\mathrm{H} 2 \mathrm{~A} & 108(3) \\ \mathrm{Mn} 1-\mathrm{O} 2-\mathrm{H} 2 \mathrm{~B} & 116(3) \\ \mathrm{H} 2 \mathrm{~A}-\mathrm{O} 2-\mathrm{H} 2 \mathrm{~B} & 110(4) \\ \mathrm{C} 1-\mathrm{C} 2-\mathrm{H} 2 & 119.7 \\ \mathrm{C} 3-\mathrm{C} 2-\mathrm{C} 1 & 120.5(3) \\ \mathrm{C} 3-\mathrm{C} 2-\mathrm{H} 2 & 119.7 \\ \mathrm{C} 2-\mathrm{C} 3-\mathrm{H} 3 & 120.4 \\ \mathrm{C} 2-\mathrm{C} 3-\mathrm{C} 4 & 119.1(3) \\ \mathrm{C} 4-\mathrm{C} 3-\mathrm{H} 3 & 120.4 \\ \mathrm{C} 3-\mathrm{C} 4-\mathrm{H} 4 & 120.0 \\ \mathrm{C} 3-\mathrm{C} 4-\mathrm{C} 5 & 120.1(3) \\ \mathrm{C} 5-\mathrm{C} 4-\mathrm{H} 4 & 120.0 \\ \mathrm{~N} 1-\mathrm{C} 5-\mathrm{C} 4 & 119.1(3) \\ \mathrm{N} 1-\mathrm{C} 5-\mathrm{H} 5 & 120.5 \\ \mathrm{C} 4-\mathrm{C} 5-\mathrm{H} 5 & 120.5 \\ & \end{array}$

Symmetry codes: (i) $-x+1,-y+1,-z+1$; (ii) $-x,-y+1,-z+1$.

Hydrogen-bond geometry $\left(\AA,{ }^{\circ}\right)$

\begin{tabular}{lllll}
\hline$D-\mathrm{H} \cdots A$ & $D-\mathrm{H}$ & $\mathrm{H} \cdots A$ & $D \cdots A$ & $D-\mathrm{H} \cdots A$ \\
\hline $\mathrm{O} 2-\mathrm{H} 2 A \cdots \mathrm{Cl} 1^{\mathrm{ii}}$ & $0.83(2)$ & $2.53(2)$ & $3.348(4)$ & $168(4)$ \\
$\mathrm{O} 2-\mathrm{H} 2 B \cdots \mathrm{Cl} 1^{\mathrm{iii}}$ & $0.82(2)$ & $2.52(3)$ & $3.232(4)$ & $147(4)$ \\
\hline
\end{tabular}

Symmetry codes: (ii) $-x,-y+1,-z+1$; (iii) $x, y-1, z$.

catena-Poly[[aquachloridomanganese(II)]-di- $\mu$-chlorido-[aquachloridomanganese(II)]-bis( $\mu$-2-methylpyridine $N$ oxide)] (II)

Crystal data

$\left[\mathrm{MnCl}_{2}\left(\mathrm{C}_{6} \mathrm{H}_{7} \mathrm{NO}\right)\left(\mathrm{H}_{2} \mathrm{O}\right)\right]$

$M_{r}=252.98$

Orthorhombic, $P 2_{1} 2_{1} 2_{1}$

$a=6.862$ (2) $\AA$

$b=7.491$ (2) $\AA$

$c=18.047(5) \AA$

$V=927.7(4) \AA^{3}$
$Z=4$

$F(000)=508$

$D_{\mathrm{x}}=1.811 \mathrm{Mg} \mathrm{m}^{-3}$

Mo $K \alpha$ radiation, $\lambda=0.71075 \AA$

Cell parameters from 2686 reflections

$\theta=2.7-27.5^{\circ}$

$\mu=1.96 \mathrm{~mm}^{-1}$ 
$T=173 \mathrm{~K}$

Prism, colorless

Data collection

Rigaku XtalLab mini CCD diffractometer

$\omega$ scans

Absorption correction: multi-scan

(REQAB; Rigaku, 1998)

$T_{\text {min }}=0.563, T_{\text {max }}=0.737$

8438 measured reflections

Refinement

Refinement on $F^{2}$

Least-squares matrix: full

$R\left[F^{2}>2 \sigma\left(F^{2}\right)\right]=0.051$

$w R\left(F^{2}\right)=0.100$

$S=1.12$

2109 reflections

112 parameters

0 restraints

Primary atom site location: dual

Hydrogen site location: mixed
$0.2 \times 0.2 \times 0.1 \mathrm{~mm}$

2109 independent reflections

1800 reflections with $I>2 \sigma(I)$

$R_{\text {int }}=0.051$

$\theta_{\text {max }}=27.5^{\circ}, \theta_{\min }=2.3^{\circ}$

$h=-8 \rightarrow 8$

$k=-9 \rightarrow 9$

$l=-23 \rightarrow 23$

$\mathrm{H}$-atom parameters constrained

$w=1 /\left[\sigma^{2}\left(F_{\mathrm{o}}{ }^{2}\right)+(0.004 P)^{2}+2.3909 P\right]$

where $P=\left(F_{\mathrm{o}}{ }^{2}+2 F_{\mathrm{c}}{ }^{2}\right) / 3$

$(\Delta / \sigma)_{\max }<0.001$

$\Delta \rho_{\max }=0.95$ e $\AA^{-3}$

$\Delta \rho_{\min }=-0.73$ e $\AA^{-3}$

Absolute structure: Refined as an inversion twin.

Absolute structure parameter: 0.44 (8)

Special details

Geometry. All esds (except the esd in the dihedral angle between two 1.s. planes) are estimated using the full covariance matrix. The cell esds are taken into account individually in the estimation of esds in distances, angles and torsion angles; correlations between esds in cell parameters are only used when they are defined by crystal symmetry. An approximate (isotropic) treatment of cell esds is used for estimating esds involving 1.s. planes.

Refinement. Refined as a 2-component inversion twin.

Fractional atomic coordinates and isotropic or equivalent isotropic displacement parameters $\left(\AA^{2}\right)$

\begin{tabular}{lllll}
\hline & $x$ & $y$ & $z$ & $U_{\text {iss }} / U_{\text {eq }}$ \\
\hline Mn1 & $0.5059(2)$ & $0.73290(15)$ & $0.50137(7)$ & $0.0197(2)$ \\
C1 & $0.2537(3)$ & $0.6800(2)$ & $0.40260(8)$ & $0.0226(3)$ \\
O1 & $0.7561(8)$ & $0.7085(6)$ & $0.42855(19)$ & $0.0179(9)$ \\
N1 & $0.7503(11)$ & $0.7249(7)$ & $0.3544(2)$ & $0.0199(11)$ \\
C1 & $0.7553(14)$ & $0.5782(9)$ & $0.3112(3)$ & $0.0276(15)$ \\
C12 & $0.5309(3)$ & $0.4110(3)$ & $0.53742(11)$ & $0.0325(5)$ \\
O2 & $0.4833(8)$ & $1.0223(7)$ & $0.4655(3)$ & $0.0265(12)$ \\
H2A & 0.546895 & 1.093811 & 0.497004 & $0.040^{*}$ \\
H2B & 0.358601 & 1.056880 & 0.464095 & $0.040^{*}$ \\
C2 & $0.7532(15)$ & $0.6041(10)$ & $0.2345(3)$ & $0.0321(16)$ \\
H2 & 0.758660 & 0.503891 & 0.202342 & $0.038^{*}$ \\
C3 & $0.7436(16)$ & $0.7719(11)$ & $0.2056(4)$ & $0.0370(18)$ \\
H3 & 0.740766 & 0.787849 & 0.153377 & $0.044^{*}$ \\
C4 & $0.7378(16)$ & $0.9180(10)$ & $0.2515(4)$ & $0.0371(19)$ \\
H4 & 0.731159 & 1.035141 & 0.231495 & $0.044^{*}$ \\
C5 & $0.7418(15)$ & $0.8925(9)$ & $0.3261(3)$ & $0.0283(16)$ \\
H5 & 0.738625 & 0.992359 & 0.358479 & $0.034^{*}$ \\
C6 & $0.7629(17)$ & $0.4059(10)$ & $0.3484(4)$ & $0.040(2)$
\end{tabular}


supporting information

\begin{tabular}{lllll} 
H6A & 0.757534 & 0.310158 & 0.311520 & $0.048^{*}$ \\
H6B & 0.651845 & 0.395059 & 0.382265 & $0.048^{*}$ \\
H6C & 0.884583 & 0.396660 & 0.376629 & $0.048^{*}$ \\
\hline
\end{tabular}

Atomic displacement parameters $\left(\AA^{2}\right)$

\begin{tabular}{lllllll}
\hline & $U^{11}$ & $U^{22}$ & $U^{33}$ & $U^{12}$ & $U^{13}$ & $U^{23}$ \\
\hline Mn1 & $0.0171(4)$ & $0.0266(5)$ & $0.0154(4)$ & $-0.0028(5)$ & $0.0017(4)$ & $-0.0008(4)$ \\
C11 & $0.0212(7)$ & $0.0300(9)$ & $0.0167(6)$ & $0.0003(10)$ & $0.0004(8)$ & $-0.0069(6)$ \\
O1 & $0.020(2)$ & $0.025(3)$ & $0.0089(16)$ & $-0.005(3)$ & $-0.002(2)$ & $-0.0013(16)$ \\
N1 & $0.019(2)$ & $0.029(3)$ & $0.012(2)$ & $0.002(3)$ & $0.002(3)$ & $-0.001(2)$ \\
C1 & $0.028(4)$ & $0.028(4)$ & $0.026(3)$ & $0.003(5)$ & $-0.001(4)$ & $-0.005(3)$ \\
C12 & $0.0377(12)$ & $0.0234(10)$ & $0.0365(11)$ & $-0.0021(10)$ & $0.0037(9)$ & $0.0050(8)$ \\
O2 & $0.015(3)$ & $0.048(3)$ & $0.016(2)$ & $-0.009(3)$ & $0.002(2)$ & $-0.003(2)$ \\
C2 & $0.036(4)$ & $0.046(5)$ & $0.015(3)$ & $0.007(6)$ & $0.002(4)$ & $-0.004(3)$ \\
C3 & $0.048(5)$ & $0.046(5)$ & $0.017(3)$ & $0.004(6)$ & $0.001(4)$ & $0.001(3)$ \\
C4 & $0.060(5)$ & $0.027(4)$ & $0.024(4)$ & $0.002(6)$ & $0.003(5)$ & $0.005(3)$ \\
C5 & $0.042(4)$ & $0.026(4)$ & $0.017(3)$ & $0.007(5)$ & $0.004(4)$ & $-0.002(3)$ \\
C6 & $0.061(6)$ & $0.029(4)$ & $0.029(4)$ & $0.007(6)$ & $0.001(5)$ & $0.000(3)$ \\
\hline
\end{tabular}

Geometric parameters $\left(\AA,{ }^{\circ}\right)$

\begin{tabular}{|c|c|c|c|}
\hline $\mathrm{Mn} 1-\mathrm{Cl1}^{\mathrm{i}}$ & $2.514(3)$ & $\mathrm{O} 2-\mathrm{H} 2 \mathrm{~B}$ & 0.8947 \\
\hline $\mathrm{Mn} 1-\mathrm{Cl1}$ & $2.516(4)$ & $\mathrm{C} 2-\mathrm{H} 2$ & 0.9500 \\
\hline $\mathrm{Mn} 1-\mathrm{O} 1^{\mathrm{ii}}$ & $2.174(5)$ & $\mathrm{C} 2-\mathrm{C} 3$ & $1.363(10)$ \\
\hline $\mathrm{Mn} 1-\mathrm{O} 1$ & $2.171(6)$ & $\mathrm{C} 3-\mathrm{H} 3$ & 0.9500 \\
\hline $\mathrm{Mn} 1-\mathrm{Cl} 2$ & $2.503(4)$ & $\mathrm{C} 3-\mathrm{C} 4$ & $1.374(10)$ \\
\hline $\mathrm{Mn} 1-\mathrm{O} 2$ & $2.268(6)$ & $\mathrm{C} 4-\mathrm{H} 4$ & 0.9500 \\
\hline $\mathrm{O} 1-\mathrm{N} 1$ & $1.345(6)$ & $\mathrm{C} 4-\mathrm{C} 5$ & $1.360(9)$ \\
\hline $\mathrm{N} 1-\mathrm{C} 1$ & $1.348(8)$ & $\mathrm{C} 5-\mathrm{H} 5$ & 0.9500 \\
\hline $\mathrm{N} 1-\mathrm{C} 5$ & $1.357(8)$ & C6-H6A & 0.9800 \\
\hline $\mathrm{C} 1-\mathrm{C} 2$ & $1.397(9)$ & $\mathrm{C} 6-\mathrm{H} 6 \mathrm{~B}$ & 0.9800 \\
\hline $\mathrm{C} 1-\mathrm{C} 6$ & $1.456(10)$ & C6-H6C & 0.9800 \\
\hline $\mathrm{O} 2-\mathrm{H} 2 \mathrm{~A}$ & 0.8951 & & \\
\hline $\mathrm{C} 11^{\mathrm{i}}-\mathrm{Mn} 1-\mathrm{Cl1}$ & $174.01(5)$ & $\mathrm{N} 1-\mathrm{C} 1-\mathrm{C} 6$ & $117.1(6)$ \\
\hline 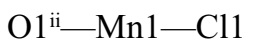 & $84.38(18)$ & $\mathrm{C} 2-\mathrm{C} 1-\mathrm{C} 6$ & $125.5(7)$ \\
\hline $\mathrm{O} 1-\mathrm{Mn} 1-\mathrm{C} 11^{\mathrm{i}}$ & 84.49 (18) & $\mathrm{Mn} 1-\mathrm{O} 2-\mathrm{H} 2 \mathrm{~A}$ & 110.9 \\
\hline $\mathrm{O} 1^{\mathrm{ii}-}-\mathrm{Mn} 1-\mathrm{C} 11^{\mathrm{i}}$ & $94.57(18)$ & $\mathrm{Mn} 1-\mathrm{O} 2-\mathrm{H} 2 \mathrm{~B}$ & 110.5 \\
\hline $\mathrm{O} 1-\mathrm{Mn} 1-\mathrm{Cl} 1$ & $95.84(18)$ & $\mathrm{H} 2 \mathrm{~A}-\mathrm{O} 2-\mathrm{H} 2 \mathrm{~B}$ & 108.1 \\
\hline $\mathrm{O} 1-\mathrm{Mn} 1-\mathrm{O} 1^{\mathrm{ii}}$ & $173.11(6)$ & $\mathrm{C} 1-\mathrm{C} 2-\mathrm{H} 2$ & 119.7 \\
\hline $\mathrm{O} 1-\mathrm{Mn} 1-\mathrm{Cl} 2$ & $91.26(14)$ & $\mathrm{C} 3-\mathrm{C} 2-\mathrm{C} 1$ & $120.5(7)$ \\
\hline 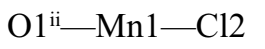 & $95.58(14)$ & $\mathrm{C} 3-\mathrm{C} 2-\mathrm{H} 2$ & 119.7 \\
\hline 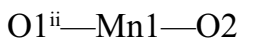 & 85.39 (19) & $\mathrm{C} 2-\mathrm{C} 3-\mathrm{H} 3$ & 119.8 \\
\hline $\mathrm{O} 1-\mathrm{Mn} 1-\mathrm{O} 2$ & 87.78 (19) & $\mathrm{C} 2-\mathrm{C} 3-\mathrm{C} 4$ & $120.3(6)$ \\
\hline $\mathrm{C} 12-\mathrm{Mn} 1-\mathrm{C} 11^{\mathrm{i}}$ & $91.40(9)$ & $\mathrm{C} 4-\mathrm{C} 3-\mathrm{H} 3$ & 119.8 \\
\hline $\mathrm{C} 12-\mathrm{Mn} 1-\mathrm{Cl} 1$ & $94.57(9)$ & $\mathrm{C} 3-\mathrm{C} 4-\mathrm{H} 4$ & 120.5 \\
\hline $\mathrm{O} 2-\mathrm{Mn} 1-\mathrm{Cl} 1$ & $84.34(16)$ & $\mathrm{C} 5-\mathrm{C} 4-\mathrm{C} 3$ & $119.0(7)$ \\
\hline
\end{tabular}




$\begin{array}{llll}\mathrm{O} 2-\mathrm{Mn} 1-\mathrm{Cl1} & \mathrm{C} 5-\mathrm{C} 4-\mathrm{H} 4 & 120.5 \\ \mathrm{O} 2-\mathrm{Mn} 1-\mathrm{Cl} 2 & 89.71(16) & \mathrm{N} 1-\mathrm{C} 5-\mathrm{C} 4 & 120.3(6) \\ \mathrm{Mn} 11^{\mathrm{i}}-\mathrm{C} 1-\mathrm{Mn} 1 & 178.46(15) & \mathrm{N} 1-\mathrm{C} 5-\mathrm{H} 5 & 119.9 \\ \mathrm{Mn} 1-\mathrm{O} 1-\mathrm{Mn}^{\mathrm{i}} & 86.32(13) & \mathrm{C} 4-\mathrm{C} 5-\mathrm{H} 5 & 119.9 \\ \mathrm{~N} 1-\mathrm{O} 1-\mathrm{Mn} 1^{\mathrm{i}} & 104.73(19) & \mathrm{C} 1-\mathrm{C} 6-\mathrm{H} 6 \mathrm{~A} & 109.5 \\ \mathrm{~N} 1-\mathrm{O} 1-\mathrm{Mn} 1 & 125.7(5) & \mathrm{C} 1-\mathrm{C} 6-\mathrm{H} 6 \mathrm{~B} & 109.5 \\ \mathrm{O} 1-\mathrm{N} 1-\mathrm{C} 1 & 124.9(5) & \mathrm{C} 1-\mathrm{C} 6-\mathrm{H} 6 \mathrm{C} & 109.5 \\ \mathrm{O} 1-\mathrm{N} 1-\mathrm{C} 5 & 120.1(5) & \mathrm{H} 6 \mathrm{~A}-\mathrm{C} 6-\mathrm{H} 6 \mathrm{~B} & 109.5 \\ \mathrm{C} 1-\mathrm{N} 1-\mathrm{C} 5 & 117.4(5) & \mathrm{H} 6 \mathrm{~B}-\mathrm{C} 6-\mathrm{H} 6 \mathrm{C} & 109.5 \\ \mathrm{~N} 1-\mathrm{C} 1-\mathrm{C} 2 & 122.5(5) & \mathrm{C} 1-\mathrm{N} 1-\mathrm{C} 5-\mathrm{C} 4 & 109.5 \\ & 117.4(7) & \mathrm{C} 1-\mathrm{C} 2-\mathrm{C} 3-\mathrm{C} 4 & \\ \mathrm{Mn} 1-\mathrm{O} 1-\mathrm{N} 1-\mathrm{C} 1 & & \mathrm{C} 2-\mathrm{C} 3-\mathrm{C} 4-\mathrm{C} 5 & 0.7(15) \\ \mathrm{Mn} 1{ }^{\mathrm{i}}-\mathrm{O} 1-\mathrm{N} 1-\mathrm{C} 1 & -102.4(8) & \mathrm{C} 3-\mathrm{C} 4-\mathrm{C} 5-\mathrm{N} 1 & 0.0(17) \\ \mathrm{Mn} 1-\mathrm{O} 1-\mathrm{N} 1-\mathrm{C} 5 & 105.6(8) & \mathrm{C} 5-\mathrm{N} 1-\mathrm{C} 1-\mathrm{C} 2 & -0.3(17) \\ \mathrm{Mn} 1-\mathrm{O} 1-\mathrm{N} 1-\mathrm{C} 5 & 78.4(9) & \mathrm{C} 5-\mathrm{N} 1-\mathrm{C} 1-\mathrm{C} 6 & 0.6(14) \\ \mathrm{O} 1-\mathrm{N} 1-\mathrm{C} 1-\mathrm{C} 2 & -73.7(9) & \mathrm{C} 6-\mathrm{C} 1-\mathrm{C} 2-\mathrm{C} 3 & -179.6(10) \\ \mathrm{O} 1-\mathrm{N} 1-\mathrm{C} 1-\mathrm{C} 6 & -178.6(8) & & 179.2(11) \\ \mathrm{O} 1-\mathrm{N} 1-\mathrm{C} 5-\mathrm{C} 4 & 1.2(13) & & \\ \mathrm{N} 1-\mathrm{C} 1-\mathrm{C} 2-\mathrm{C} 3 & 179.3(9) & & \end{array}$

Symmetry codes: (i) $x+1 / 2,-y+3 / 2,-z+1$; (ii) $x-1 / 2,-y+3 / 2,-z+1$.

Hydrogen-bond geometry $\left(\AA,{ }^{\circ}\right)$

\begin{tabular}{lllll}
\hline$D-\mathrm{H} \cdots A$ & $D-\mathrm{H}$ & $\mathrm{H} \cdots A$ & $D \cdots A$ & $D-\mathrm{H} \cdots A$ \\
\hline $\mathrm{O} 2-\mathrm{H} 2 A \cdots \mathrm{Cl} 2^{\mathrm{iii}}$ & 0.90 & 2.49 & $3.205(7)$ & 137 \\
$\mathrm{O} 2-\mathrm{H} 2 B \cdots \mathrm{Cl} 2^{\mathrm{ii}}$ & 0.89 & 2.26 & $3.145(7)$ & 169 \\
\hline
\end{tabular}

Symmetry codes: (ii) $x-1 / 2,-y+3 / 2,-z+1$; (iii) $x, y+1, z$.

Bis( $\mu$-3-methylpyridine $\mathrm{N}$-oxide)bis[diaquadichloridomanganese(II)] (III)

Crystal data

$\left[\mathrm{Mn}_{2} \mathrm{Cl}_{4}\left(\mathrm{C}_{6} \mathrm{H}_{7} \mathrm{NO}\right)_{2}\left(\mathrm{H}_{2} \mathrm{O}\right)_{4}\right]$

$M_{r}=541.99$

Triclinic, $P \overline{1}$

$a=7.902(7) \AA$

$b=8.026(7) \AA$

$c=9.893(8) \AA$

$\alpha=98.033(1)^{\circ}$

$\beta=99.272(7)^{\circ}$

$\gamma=113.634(11)^{\circ}$

$V=552.6(8) \AA^{3}$

\section{Data collection}

Rigaku XtalLab mini CCD diffractometer

$\omega$ scans

Absorption correction: multi-scan

(REQAB; Rigaku, 1998)

$T_{\min }=0.482, T_{\max }=0.630$

5837 measured reflections
$Z=1$

$F(000)=274$

$D_{\mathrm{x}}=1.629 \mathrm{Mg} \mathrm{m}^{-3}$

Mo $K \alpha$ radiation, $\lambda=0.71073 \AA$

Cell parameters from 5886 reflections

$\theta=2.8-27.5^{\circ}$

$\mu=1.65 \mathrm{~mm}^{-1}$

$T=173 \mathrm{~K}$

Prism, clear brown

$0.85 \times 0.5 \times 0.28 \mathrm{~mm}$

2553 independent reflections

2375 reflections with $I>2 \sigma(I)$

$R_{\text {int }}=0.072$

$\theta_{\max }=27.6^{\circ}, \theta_{\min }=2.8^{\circ}$

$h=-10 \rightarrow 10$

$k=-10 \rightarrow 10$

$l=-12 \rightarrow 12$ 


\section{Refinement}

Refinement on $F^{2}$

Least-squares matrix: full

$R\left[F^{2}>2 \sigma\left(F^{2}\right)\right]=0.031$

$w R\left(F^{2}\right)=0.087$

$S=1.07$

2553 reflections

135 parameters

4 restraints

Primary atom site location: dual

Special details

Geometry. All esds (except the esd in the dihedral angle between two 1.s. planes) are estimated using the full covariance matrix. The cell esds are taken into account individually in the estimation of esds in distances, angles and torsion angles; correlations between esds in cell parameters are only used when they are defined by crystal symmetry. An approximate (isotropic) treatment of cell esds is used for estimating esds involving l.s. planes.

Fractional atomic coordinates and isotropic or equivalent isotropic displacement parameters $\left(\AA^{2}\right)$

\begin{tabular}{lllll}
\hline & $x$ & $y$ & $z$ & $U_{\text {iso }} * / U_{\text {eq }}$ \\
\hline Mn1 & $0.27031(3)$ & $0.40104(3)$ & $0.53553(2)$ & $0.02936(11)$ \\
C11 & $0.14922(6)$ & $0.48607(7)$ & $0.73553(4)$ & $0.04342(14)$ \\
C12 & $0.32299(6)$ & $0.13672(6)$ & $0.60668(6)$ & $0.04544(15)$ \\
O1 & $0.56562(15)$ & $0.60090(16)$ & $0.62683(12)$ & $0.0345(3)$ \\
O2 & $0.2605(2)$ & $0.6467(2)$ & $0.45620(16)$ & $0.0443(3)$ \\
H2A & $0.355(3)$ & $0.710(3)$ & $0.436(2)$ & $0.055(7)^{*}$ \\
H2B & $0.167(3)$ & $0.639(3)$ & $0.404(2)$ & $0.062(8)^{*}$ \\
O3 & $0.00694(17)$ & $0.23434(18)$ & $0.37696(14)$ & $0.0395(3)$ \\
H3A & $-0.069(3)$ & $0.128(2)$ & $0.390(2)$ & $0.062(7)^{*}$ \\
H3B & $-0.054(4)$ & $0.288(4)$ & $0.352(3)$ & $0.081(10)^{*}$ \\
N1 & $0.62886(17)$ & $0.71302(18)$ & $0.75570(13)$ & $0.0294(3)$ \\
C1 & $0.6561(2)$ & $0.8903(2)$ & $0.77007(17)$ & $0.0342(4)$ \\
H1 & 0.631326 & 0.934009 & 0.690425 & $0.041^{*}$ \\
C2 & $0.7204(3)$ & $1.0102(3)$ & $0.90084(19)$ & $0.0415(4)$ \\
C3 & $0.7538(3)$ & $0.9400(3)$ & $1.0169(2)$ & $0.0568(6)$ \\
H3 & 0.795802 & 1.016517 & 1.106619 & $0.068^{*}$ \\
C4 & $0.7250(4)$ & $0.7571(4)$ & $0.9997(2)$ & $0.0611(6)$ \\
H4 & 0.748138 & 0.710090 & 1.077885 & $0.073^{*}$ \\
C5 & $0.6620(3)$ & $0.6431(3)$ & $0.8670(2)$ & $0.0472(5)$ \\
H5 & 0.642729 & 0.519251 & 0.854911 & $0.057^{*}$ \\
C6 & $0.7494(4)$ & $1.2087(3)$ & $0.9131(3)$ & $0.0701(7)$ \\
H6A & 0.873126 & 1.282752 & 0.900126 & $0.105^{*}$ \\
H6B & 0.739622 & 1.256760 & 1.004510 & $0.105^{*}$ \\
H6C & 0.653880 & 1.213706 & 0.842403 & $0.105^{*}$
\end{tabular}

Hydrogen site location: mixed

$\mathrm{H}$ atoms treated by a mixture of independent

and constrained refinement

$w=1 /\left[\sigma^{2}\left(F_{\mathrm{o}}^{2}\right)+(0.031 P)^{2}+0.023 P\right]$

where $P=\left(F_{\mathrm{o}}{ }^{2}+2 F_{\mathrm{c}}{ }^{2}\right) / 3$

$(\Delta / \sigma)_{\max }<0.001$

$\Delta \rho_{\max }=0.56 \mathrm{e} \AA^{-3}$

$\Delta \rho_{\min }=-0.41 \mathrm{e}^{-3}$ 


\begin{tabular}{lllllll}
$\mathrm{C} 12$ & $0.0436(3)$ & $0.0349(3)$ & $0.0596(3)$ & $0.0137(2)$ & $0.0151(2)$ & $0.0236(2)$ \\
$\mathrm{O} 1$ & $0.0326(6)$ & $0.0310(6)$ & $0.0313(6)$ & $0.0074(5)$ & $0.0091(5)$ & $-0.0010(5)$ \\
$\mathrm{O} 2$ & $0.0389(7)$ & $0.0414(7)$ & $0.0584(9)$ & $0.0185(6)$ & $0.0137(6)$ & $0.0231(7)$ \\
$\mathrm{O} 3$ & $0.0339(6)$ & $0.0322(7)$ & $0.0438(7)$ & $0.0079(5)$ & $0.0039(5)$ & $0.0080(6)$ \\
$\mathrm{N} 1$ & $0.0295(6)$ & $0.0293(7)$ & $0.0271(6)$ & $0.0107(5)$ & $0.0061(5)$ & $0.0062(5)$ \\
$\mathrm{C} 1$ & $0.0443(9)$ & $0.0307(8)$ & $0.0273(7)$ & $0.0159(7)$ & $0.0082(6)$ & $0.0067(6)$ \\
$\mathrm{C} 2$ & $0.0484(10)$ & $0.0363(9)$ & $0.0336(8)$ & $0.0138(8)$ & $0.0112(7)$ & $0.0016(7)$ \\
$\mathrm{C} 3$ & $0.0674(13)$ & $0.0598(13)$ & $0.0262(9)$ & $0.0158(11)$ & $0.0049(9)$ & $0.0014(9)$ \\
$\mathrm{C} 4$ & $0.0766(14)$ & $0.0687(15)$ & $0.0355(10)$ & $0.0289(12)$ & $0.0028(10)$ & $0.0246(10)$ \\
$\mathrm{C} 5$ & $0.0591(11)$ & $0.0424(10)$ & $0.0425(10)$ & $0.0236(9)$ & $0.0064(9)$ & $0.0186(9)$ \\
$\mathrm{C} 6$ & $0.105(2)$ & $0.0393(11)$ & $0.0571(14)$ & $0.0257(13)$ & $0.0237(14)$ & $-0.0048(10)$ \\
\hline
\end{tabular}

Geometric parameters $\left(\AA,{ }^{\circ}\right)$

\begin{tabular}{|c|c|c|c|}
\hline $\mathrm{Mn} 1-\mathrm{Cl} 1$ & $2.4602(15)$ & $\mathrm{C} 1-\mathrm{H} 1$ & 0.9300 \\
\hline $\mathrm{Mn} 1-\mathrm{Cl} 2$ & $2.4900(19)$ & $\mathrm{C} 1-\mathrm{C} 2$ & $1.381(2)$ \\
\hline $\mathrm{Mn} 1-\mathrm{O} 1^{\mathrm{i}}$ & $2.2228(17)$ & $\mathrm{C} 2-\mathrm{C} 3$ & $1.381(3)$ \\
\hline $\mathrm{Mn} 1-\mathrm{O} 1$ & $2.1792(18)$ & $\mathrm{C} 2-\mathrm{C} 6$ & $1.500(3)$ \\
\hline $\mathrm{Mn} 1-\mathrm{O} 2$ & $2.246(2)$ & $\mathrm{C} 3-\mathrm{H} 3$ & 0.9300 \\
\hline $\mathrm{Mn} 1-\mathrm{O} 3$ & $2.1704(17)$ & $\mathrm{C} 3-\mathrm{C} 4$ & $1.373(4)$ \\
\hline $\mathrm{O} 1-\mathrm{N} 1$ & $1.3411(19)$ & $\mathrm{C} 4-\mathrm{H} 4$ & 0.9300 \\
\hline $\mathrm{O} 2-\mathrm{H} 2 \mathrm{~A}$ & $0.791(15)$ & $\mathrm{C} 4-\mathrm{C} 5$ & $1.377(3)$ \\
\hline $\mathrm{O} 2-\mathrm{H} 2 \mathrm{~B}$ & $0.802(16)$ & $\mathrm{C} 5-\mathrm{H} 5$ & 0.9300 \\
\hline $\mathrm{O} 3-\mathrm{H} 3 \mathrm{~A}$ & $0.863(16)$ & $\mathrm{C} 6-\mathrm{H} 6 \mathrm{~A}$ & 0.9600 \\
\hline $\mathrm{O} 3-\mathrm{H} 3 \mathrm{~B}$ & 0.795 (17) & $\mathrm{C} 6-\mathrm{H} 6 \mathrm{~B}$ & 0.9600 \\
\hline $\mathrm{N} 1-\mathrm{C} 1$ & $1.334(2)$ & $\mathrm{C} 6-\mathrm{H} 6 \mathrm{C}$ & 0.9600 \\
\hline $\mathrm{N} 1-\mathrm{C} 5$ & $1.340(2)$ & & \\
\hline $\mathrm{Cl} 1-\mathrm{Mn} 1-\mathrm{Cl} 2$ & $98.31(4)$ & $\mathrm{C} 1-\mathrm{N} 1-\mathrm{O} 1$ & $119.45(13)$ \\
\hline $\mathrm{O} 1-\mathrm{Mn} 1-\mathrm{C} 11$ & $95.44(6)$ & $\mathrm{C} 1-\mathrm{N} 1-\mathrm{C} 5$ & $121.69(16)$ \\
\hline $\mathrm{O} 1^{\mathrm{i}}-\mathrm{Mn} 1-\mathrm{Cl1}$ & $165.45(4)$ & $\mathrm{C} 5-\mathrm{N} 1-\mathrm{O} 1$ & $118.86(16)$ \\
\hline $\mathrm{O} 1^{\mathrm{i}}-\mathrm{Mn} 1-\mathrm{Cl} 2$ & $89.66(5)$ & $\mathrm{N} 1-\mathrm{C} 1-\mathrm{H} 1$ & 119.3 \\
\hline $\mathrm{O} 1-\mathrm{Mn} 1-\mathrm{Cl} 2$ & $93.11(7)$ & $\mathrm{N} 1-\mathrm{C} 1-\mathrm{C} 2$ & $121.31(16)$ \\
\hline $\mathrm{O} 1-\mathrm{Mn} 1-\mathrm{O} 1^{\mathrm{i}}$ & $71.87(7)$ & $\mathrm{C} 2-\mathrm{C} 1-\mathrm{H} 1$ & 119.3 \\
\hline $\mathrm{O} 1-\mathrm{Mn} 1-\mathrm{O} 2$ & $82.07(6)$ & $\mathrm{C} 1-\mathrm{C} 2-\mathrm{C} 3$ & $117.8(2)$ \\
\hline $\mathrm{O} 1-\mathrm{Mn} 1-\mathrm{O} 2$ & $81.64(7)$ & $\mathrm{C} 1-\mathrm{C} 2-\mathrm{C} 6$ & 119.87 (19) \\
\hline $\mathrm{O} 2-\mathrm{Mn} 1-\mathrm{Cl} 1$ & $89.20(6)$ & $\mathrm{C} 3-\mathrm{C} 2-\mathrm{C} 6$ & $122.4(2)$ \\
\hline $\mathrm{O} 2-\mathrm{Mn} 1-\mathrm{Cl} 2$ & $171.24(4)$ & $\mathrm{C} 2-\mathrm{C} 3-\mathrm{H} 3$ & 120.0 \\
\hline $\mathrm{O} 3-\mathrm{Mn} 1-\mathrm{Cl} 1$ & $101.10(7)$ & $\mathrm{C} 4-\mathrm{C} 3-\mathrm{C} 2$ & 119.99 (19) \\
\hline $\mathrm{O} 3-\mathrm{Mn} 1-\mathrm{Cl} 2$ & $97.13(6)$ & $\mathrm{C} 4-\mathrm{C} 3-\mathrm{H} 3$ & 120.0 \\
\hline $\mathrm{O} 3-\mathrm{Mn} 1-\mathrm{O} 1^{\mathrm{i}}$ & $89.88(8)$ & $\mathrm{C} 3-\mathrm{C} 4-\mathrm{H} 4$ & 119.9 \\
\hline $\mathrm{O} 3-\mathrm{Mn} 1-\mathrm{O} 1$ & $159.02(5)$ & $\mathrm{C} 3-\mathrm{C} 4-\mathrm{C} 5$ & $120.16(18)$ \\
\hline $\mathrm{O} 3-\mathrm{Mn} 1-\mathrm{O} 2$ & $85.76(7)$ & $\mathrm{C} 5-\mathrm{C} 4-\mathrm{H} 4$ & 119.9 \\
\hline $\mathrm{Mn} 1-\mathrm{O} 1-\mathrm{Mn} 1^{\mathrm{i}}$ & $108.13(7)$ & $\mathrm{N} 1-\mathrm{C} 5-\mathrm{C} 4$ & $119.10(19)$ \\
\hline $\mathrm{N} 1-\mathrm{O} 1-\mathrm{Mn} 1$ & $124.29(9)$ & $\mathrm{N} 1-\mathrm{C} 5-\mathrm{H} 5$ & 120.5 \\
\hline $\mathrm{N} 1-\mathrm{O} 1-\mathrm{Mn} 1^{\mathrm{i}}$ & $127.41(10)$ & $\mathrm{C} 4-\mathrm{C} 5-\mathrm{H} 5$ & 120.5 \\
\hline $\mathrm{Mn} 1-\mathrm{O} 2-\mathrm{H} 2 \mathrm{~A}$ & $114.4(17)$ & $\mathrm{C} 2-\mathrm{C} 6-\mathrm{H} 6 \mathrm{~A}$ & 109.5 \\
\hline $\mathrm{Mn} 1-\mathrm{O} 2-\mathrm{H} 2 \mathrm{~B}$ & $121.9(18)$ & $\mathrm{C} 2-\mathrm{C} 6-\mathrm{H} 6 \mathrm{~B}$ & 109.5 \\
\hline
\end{tabular}


supporting information

$\begin{array}{llll}\mathrm{H} 2 \mathrm{~A}-\mathrm{O} 2-\mathrm{H} 2 \mathrm{~B} & 112(2) & \mathrm{C} 2-\mathrm{C} 6-\mathrm{H} 6 \mathrm{C} & 109.5 \\ \mathrm{Mn} 1-\mathrm{O} 3-\mathrm{H} 3 \mathrm{~A} & 118.3(16) & \mathrm{H} 6 \mathrm{~A}-\mathrm{C} 6-\mathrm{H} 6 \mathrm{~B} & 109.5 \\ \mathrm{Mn} 1-\mathrm{O} 3-\mathrm{H} 3 \mathrm{~B} & 116(2) & \mathrm{H} 6 \mathrm{~A}-\mathrm{C} 6-\mathrm{H} 6 \mathrm{C} & 109.5 \\ \mathrm{H} 3 \mathrm{~A}-\mathrm{O} 3-\mathrm{H} 3 \mathrm{~B} & 109(3) & \mathrm{H} 6 \mathrm{~B}-\mathrm{C} 6-\mathrm{H} 6 \mathrm{C} & 109.5\end{array}$

Symmetry code: (i) $-x+1,-y+1,-z+1$.

Hydrogen-bond geometry $\left(A,{ }^{\circ}\right)$

\begin{tabular}{lllll}
\hline$D-\mathrm{H} \cdots A$ & $D-\mathrm{H}$ & $\mathrm{H} \cdots A$ & $D \cdots A$ & $D-\mathrm{H} \cdots A$ \\
\hline $\mathrm{O} 2-\mathrm{H} 2 B \cdots \mathrm{Cl} 1^{\mathrm{ii}}$ & $0.80(2)$ & $2.38(2)$ & $3.147(3)$ & $161(2)$ \\
$\mathrm{O} 3-\mathrm{H} 3 A \cdots \mathrm{Cl} 2^{\mathrm{iii}}$ & $0.86(2)$ & $2.28(2)$ & $3.120(2)$ & $167(2)$
\end{tabular}

Symmetry codes: (ii) $-x,-y+1,-z+1$; (iii) $-x,-y,-z+1$. 